\title{
Implementation of Oximetry Sensors for Cardiovascular Load Monitoring when Physical Exercise
}

\section{Dhodit Rengga Tisna, *M. Udin Harun Al Rasyid, Sritrusta Sukaridhoto}

\author{
Politeknik Elektronika Negeri Surabaya (PENS), Indonesia \\ Email : dhotrengga@gmail.com, udinharun@pens.ac.id, dhoto@pens.ac.id \\ *Corresponding Author: udinharun@pens.ac.id
}

Received February 7, 2020; Revised April 19, 2020; Accepted May 11, 2020

\begin{abstract}
The performance condition of an athlete must always be maintained, one way to maintain that performance is by training. Each individual has different abilities and physiological responses in receiving the portion of the exercise. Physical exercise that exceeds the body's ability can worsen the condition of the athlete itself which can result in excessive fatigue (overtraining) or can even result in injury. Therefore a system is needed to monitor the condition of the physiological response when given the intensity of the training load so that the portion of the training provided provides positive benefits for the athlete. This system was developed using an oximetry sensor, microcontroller and wifi module ESP8266. This system is used to collect heart rate and oxygen saturation data, then with the existing formula the heart rate value is converted to a CVL (Cardiovascular Load) value to determine the level of fatigue in athletes when given the intensity of the training load. By using a web-based application, measurement data is displayed in realtime to make it easier to see the results of monitoring. From the experimental results the system can monitor changes in the physiological condition of the athlete when given the intensity of the training load. Finally, the developed system can collect athlete's physiological data, and can store the data in a database and display it in a web application.
\end{abstract}

Keywords: monitoring, heart rate, oxygen saturation, CVL, overtraining.

\section{INTRODUCTION}

Athlete's achievement is influenced by the training that is always done consistently and continuously. Training is the main way for keeping and increasing the athlete's performance. By doing training consistently and continuously, it hopes that the fitness Condition off the athlete can be kept, so the athlete can reach their best performance when they are on the match. Cardiovascular Fitness is one of the sign for the healthiness that can be used in all age and gender. without including healthiness condition notes that has been taken before [1]. The cardiovascular durability directs about the 
ability of the lungs and heart for pumping the oxygen inside the blood trough all muscles as long as people doing physical activities continuously. With oxygen supplies and circulations to all parts of the body equally, cells will work very well and produces energy.

The performance of the athlete's physical condition in responding to the portion of the exercise can be assessed by the difference in the negative results of the training, fatigue and the positive results of the training, namely fitness. The ideal training menu is by giving the proper training load to reach maximal performance [2]. Monitoring training load is very important to improve performance, maintain fitness and prevent overtraining, which is a condition where a person who is given training experiences excessive fatigue. Monitoring during training is needed to determine a person's physiological response to the burden of training provided [3], with effective monitoring can provide important feedback to help in planning and training speriodization, optimizing physical conditions, and avoiding more serious injuries $[4,5]$.

Changes in the autonomic nervous system (ANS) can be used to conclude the status of training. ANS has correlation with physiologic system inside the body, valuing responses about the changes of physiologic condition shows the ability of adapting with the exercise load given. Heart rate can be used as the indicator status of training as the result of the measurement noninvasive from ANS performances [6]. Heart rate is the indicator that has direct responses to the condition that is happened in cardiovascular, so it can be used for knowing the athlete's responses and adaptations to the training and loads given. By knowing the body's responses to the loads given, the trainer can choose the proper training menu with athlete's physics capacity. So the training that is given do not give excessive fatigue and keep the fitness. [7]. The increasing of heart rate as long as physical activity can be defined as the increase of metabolism that produce of energy as the result of chemical reaction inside the body [8]. At the time of exercise muscle activity will increase, this is what causes an increase in heat production in the body as a result of the lack of metabolic efficiency in providing energy for muscle strength [9]. Many devices to measure the condition of the user's heart rate during exercise, the equipment will record the condition of the user's heartbeat, but the available equipment does not monitor the cardiovascular load experienced during exercise. Monitoring cardiovascular conditions is very likely to be needed to maintain the condition while exercising does not exceed body limits, so the risk of injury can be avoided.

At this time monitoring on athletes is still manual with writing on paper, and there is no database as a place to store physiological data from training results. Monitoring existing physiological conditions is limited to recording, without knowing at what level physiological conditions are experienced and there is no equipment to monitor the level of cardiovascular burden that occurs when training is given. In this study, we propose a web-based platform for real-time monitoring of physiological responses of athletes 
when given physical load during training and provide information about the physiological level experienced with the aim of avoiding excessive exercise and serious injury. Physiological conditions that are monitored are heart rate and oxygen saturation, then also monitor the level of CVL (Cardiovascular Load) conditions to determine the training load on the body and the level of fatigue experienced. By monitoring physiological conditions and the training load experienced during training, it is hoped that athletes do not experience excessive fatigue and fitness can be maintained. The sensor used in this study is the MAX30100 pulse oximetry sensor which detects the heart rate and oxygen saturation, then the heart rate when given training load is converted into an existing formula into a CVL value to determine the level of fatigue. Then monitoring data is displayed on the monitoring website and monitoring data is stored in a database.

\section{RELATED WORKS}

There are several studies that have been done before, in terms of monitoring the health and physiological conditions of a person. Zulkifli et al. [10] proposed research for monitoring heart rate used for various sports. Zulkifli proposed monitoring heart rate using the Garmin Heart Rate. Sending sensor data using the Xbee module is applied in this study and by using the wirelees mesh network topology it is possible to monitor the condition of several athletes simultaneously. The monitoring results sent to the coordinator of several different Garmin Heart Rate will then be displayed using LabView software.

Malhi et al. [11] proposed the design and development of non-invasive devices that are used for monitoring physiological parameters using transmission media in the form of Zigbee modules. This system is used to monitor physiological parameters in the form of heart rate and temperature in humans. The device developed is intended for parents who usually live alone at home, where those with weak conditions have a high risk of injury from a fall or stroke. This device uses a heart rate sensor, skin temperature and impact sensors to detect falls. The proposed device consists of a strap attached to the wrist and a device that resembles a ring attached to a finger, which allows the sensor to be mounted on the device used. An important aspect of this research is miniaturization, so that the system does not interfere with users when doing activities.

Garcia et al. [12] proposed health monitoring using multiple sensors. Garcia, proposed research to provide and evaluate systems that can facilitate communication between medical professionals / coaches and patients / athletes. The system architecture is based on distributed system components, where a person's physiological data is collected by several sensors, then the System-on-Chip platform in the form of Raspberry Pi combines information that has been distributed and makes the standardization process, then this data is sent to the Cloud for processing. This research also configures a cloud- 
based system, called ThingSpeak, to collect information from various sensors into one system, which is connected to the Internet.

Ovadia et al. [13] proposed the measurement of oxygen saturation conditions using pulse oximetry sensors in humans. The purpose of this research is to develop pulse oximetry which can be used for home care, with due regard to the aspects of cheap, portable, easy to use and without using cables. The results of the sensor measurement will then be sent using a Bluetooth connection to the smartphone application. So that the measurement results are easily understood by the user, the measurement results are presented to the user with a Graphic User Interface on the smartphone in a form resembling traffic light. Where if the red light is on then in critical condition, then for the green light indicates normal conditions, and the yellow light indicates the level of oxygen saturation in the moderate condition.

Ahmed et al. [14] proposed about monitoring important physiological parameters in humans. The purpose of this study is to monitor physiological conventions of patients based on the Internet of Things. So that by utilizing the technology of the Internet of Things is expected to make it easier for medical officers in physiological monitoring of patients. In this study the physiological parameters monitored are heart rate and body temperature. With a graphical user interface (GUI) in the form of an application on Android allows patients and doctors to access information about the physiological parameters of the body remotely.

Chen et al. [15] proposed equipment that could be used to detect heat stroke. The proposed system consists of a heart rate sensor, a body temperature sensor, and a humidity sensor to determine the condition of the surrounding environment. Then using the LoRa transmission media, the reading data will be sent to the server, for further analysis. The purpose of this system is to provide early warning of heat stroke conditions to users when exercising in a hot environment.

The idea consists of using sensors that are cheap, miniaturezed and can be used without disturbing the user with real-time feedback to the user through a web-based platform. The usage of human physiologic data is used to detect the responses and body adaptation ability in doing activities/exercise. In this research, the researcher used basic architecture in monitoring physiologic conditions; heart beat and oxygen saturation by noninvasive.

\section{ORIGINALITY}

Technology to monitor health conditions is developing rapidly at this time. In previous research $[11,12,14,16]$, they applied technology to determine a person's physiological condition where the physiological conditions measured were heart rate, temperature, and oxygen saturation. However, please note that in sports it is not only the heart rate at the time of the activity that needs to be used as a reference but, resting heart rate and 
maximum heart rate can be used as a reference to determine the condition of one's body. It is also important to note when giving training to athletes is the burden in accordance with the athlete's body condition itself [16]. CVL is also an important aspect that needs to be considered when giving training load to athletes. CVL can be used as a marker of athlete's physiological response to the training load provided [17].

Contribution from this study, we implemented real hardware using pulse oximetry sensors to detect heart rate, oxygen saturation and to determine the level of physical load or fatigue experienced during exercise. So that the next training scheduling can be done, and the most important thing is not overtraining and excessive fatigue. To get the level of physical burden or fatigue experienced during exercise, we use the CVL formula approach. Where in addition to paying attention to the heart rate during the activity, CVL calculation also pay attention to the resting heart rate and maximum heart rate according to the age of the athlete. We also provide a system for monitoring physiological conditions in real-time, through websitebased applications and applications that make it possible to monitor several athletes simultaneously. The reason why the website application was chosen is so that it can be accessed using either a smartphone or using a laptop (computer).

\section{SYSTEM DESIGN}

This section discusses the system design of equipment for monitoring the physiological condition of cardiovascular load when given a training load. The system design proposed in this study was designed by applying the Internet of Things technology. Where the system is built consisting of hardware, internet, software and storage that is on the cloud server. This hardware integrates several modules consisting of sensors, microcontrollers and wireless communication modules ESP8266. The sensors used in this study are pulse oximetry sensors that are used to detect heart rate and oxygen saturation in the blood, microcontroller as a signal processing sensor and a wifi module as a tool for sending data through wireless media. While the software consists of a rest API, a website for monitoring, with MongoDB database storage media that are all stored on the cloud server. For sending data from hardware to the web server through the media wirelles and the internet is done using the MQTT broker protocol. Data readings from the oximetry sensor in the form of heart rate and oxygen saturation signals are processed by a microcontroller to get analogue value data from the heartbeat and oxygen saturation, then using the ESP8266 wireless module the data collected by the microcontroller is sent via the internet to the MQTT broker. From MQTT broker the data is forwarded to the web server to display the value of the data on the web in realtime and the reading of the data is stored in the MongoDB database. Details of the proposed system design can be seen in Figure 1. 


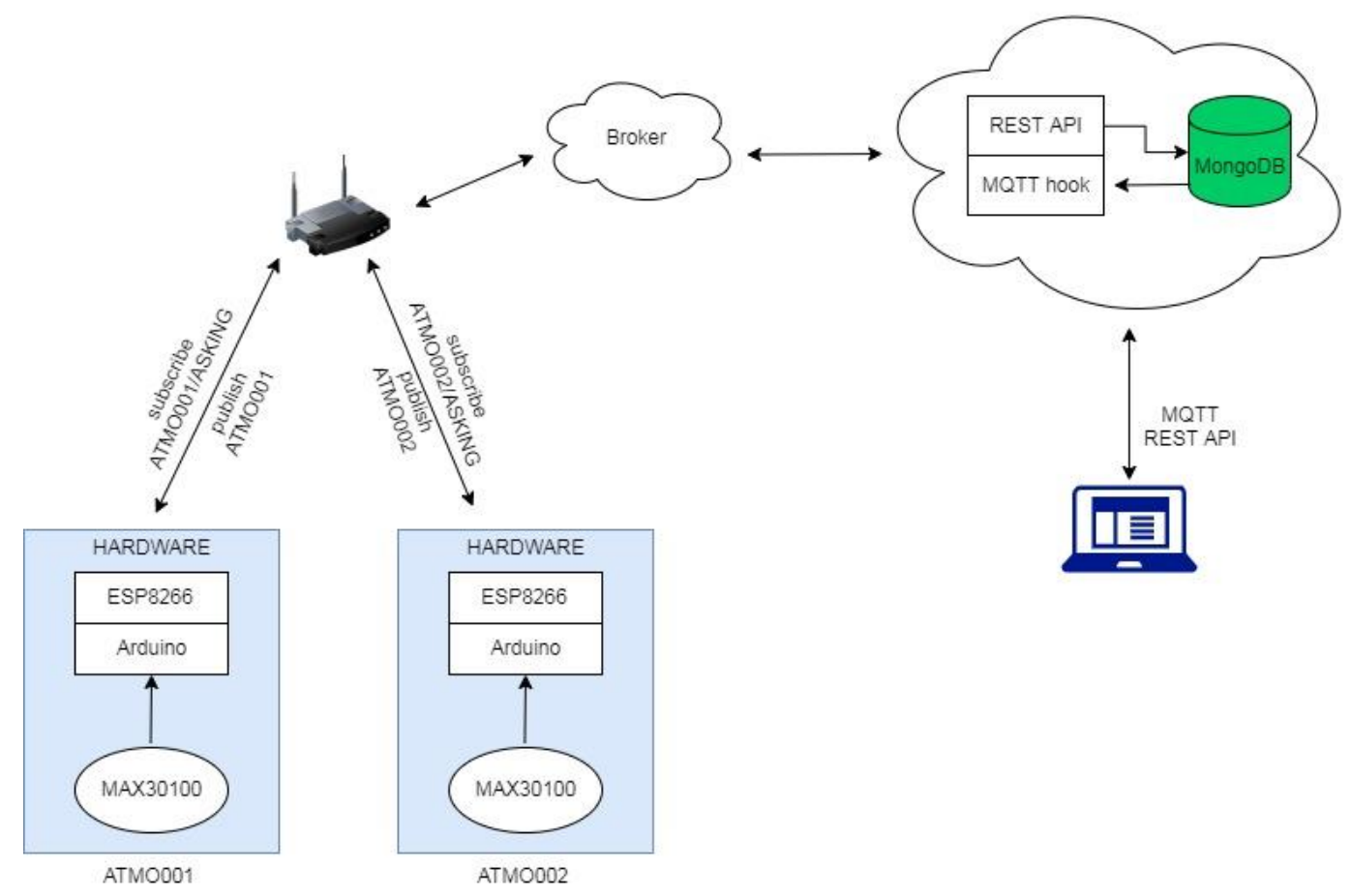

Figure 1. Monitoring system design

\subsection{Hardware System for Acquisition Data}

Acquisition Device is used to acquire data on individuals. The hardware in this study uses an oximetry sensor with the MAX-30100 type, this sensor has two variable outputs, namely heart rate and oxygen saturation. As a device for processing the input signal from the sensor used arduino nano microcontroller where the task is to change the digital signal from the sensor into an analog value so that it is easy to transmit data. Furthermore, the device for sending data from our microcontroller processing uses the ESP-8266 wireless module. In detail, the relationship between devices / modules in a series of hardware devices for sensing and processing data is shown in Figure 2.

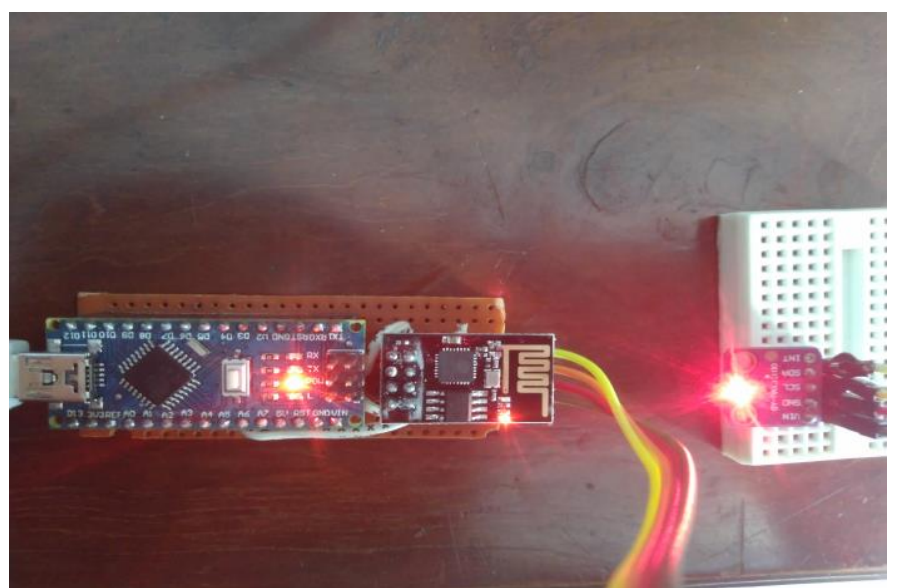

Figure 2. Hardware sensor design 


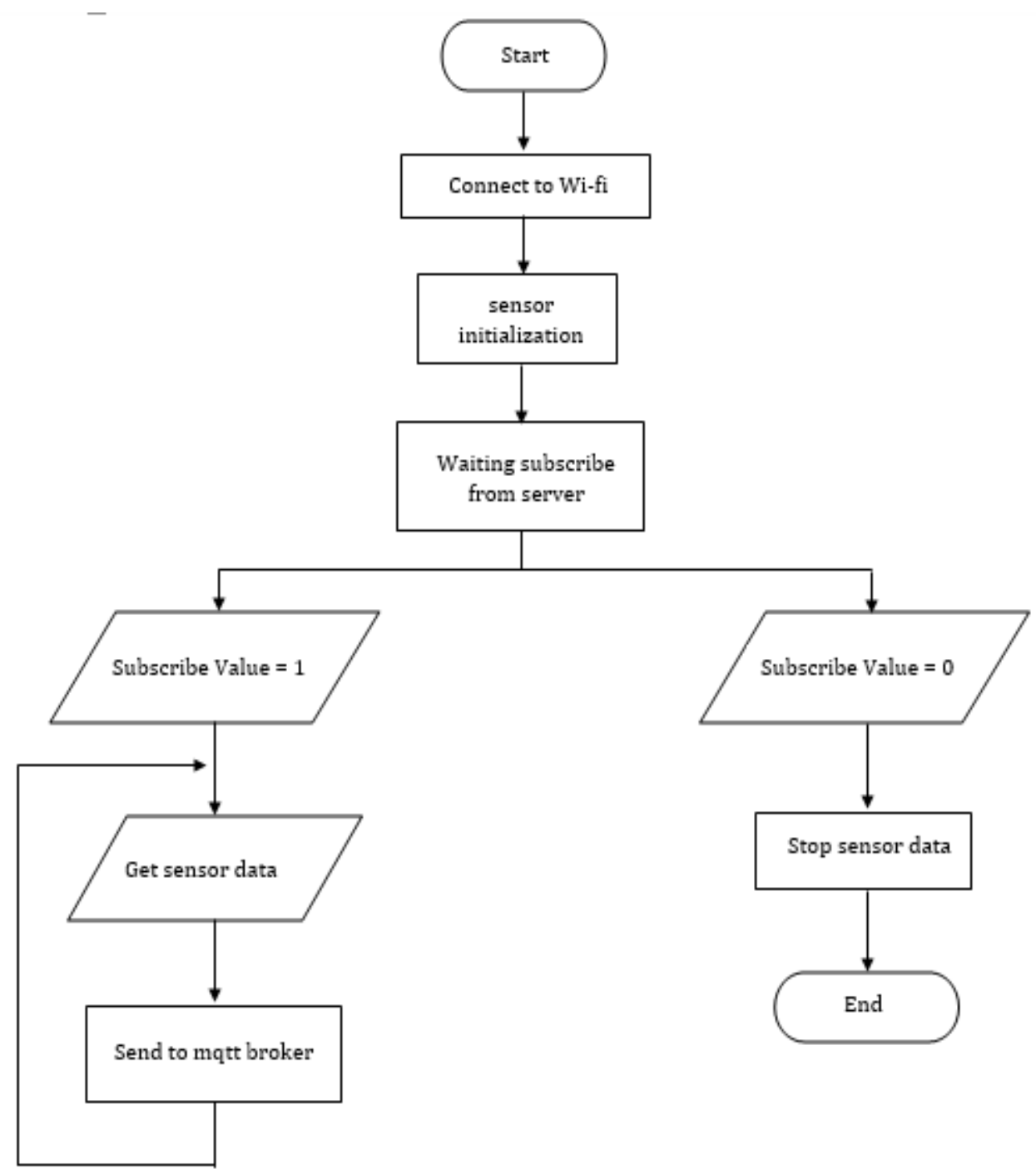

Figure 3. Flowchart sensor data acquisition and data transmission through mqtt broker

Figure 3 shows the flow of the data acquisition process on hardware installed on an individual. The hardware device must be connected to a WiFi network so you can connect with the MQTT broker. When the hardware device is connected to the wifi network, the sensor initializes the reading of the data. If the MQTT broker receives a subscription topic message with a value of 1 , it means the broker is requesting data from the device so the device will send data to the broker. However, if the broker receives a subscription topic message with a value of 0 , it means the broker will stop the process of sending data from the device to the MQTT broker. 


\subsection{Physiological Signs}

Every individual has some physiologic mark that can be measured, physiologic condition can change when the body responses to the physical activity that is done like doing sport exercise. Every individual has different responses to their own body, those are depending on the ability to adapt with external stimulation factors. By using a wireless sensor network (WSN) application, it allows sensor devices to be used or implanted into the human body so that they can be monitored remotely [18]. The sensor technology has been developed and get some advantages as the device to measure physical activity and physiologic responses to the change of vital physiologic signs when doing exercise [19]. Generally, there are five vital signs that are usually used in the professional medical field; those are heart beats, body temperature, blood pressure, respiration rate and the amount of oxygen inside the blood [20]. In the other side, Dias in [21] divides physiologic vital signs into two main field, those are medical field and the activities on the table 1.

Table 1. Physiological Signs

\begin{tabular}{|c|c|}
\hline \multicolumn{2}{|c|}{ Physiological Signs } \\
\hline \multirow{4}{*}{ Medical } & Body temperature \\
\cline { 2 - 2 } & Blood Pressure \\
\cline { 2 - 2 } & Respiration \\
\cline { 2 - 2 } & Glucose \\
\hline \multirow{3}{*}{ Activity } & Heart Rate \\
\cline { 2 - 2 } & Oxygen saturation \\
\cline { 2 - 2 } & Electrocardiogram \\
\hline
\end{tabular}

Sport has close relationship with cardiovascular system of the body, physiologic signs that influence cardiovascular system are systolic pressure, diastolic pressure, heart rate and blood oxygen like on [22]. Based on the literature about physiological that has been told before, so we took two signs, physiologic heart and oxygen saturation which we assumed corresponding with activities and cardiovascular system.

\subsection{Heart Rate}

The heart is the most vital organ for the body. Heart rate pressure is influenced by age, physical activities such as exercise, air temperature, emotional level, weight and consumption of certain drugs. Heart rate is one of the physical aspects that can affect an athlete. Heart rate is one of the vital signs that can be used to detect an individual's condition if in an abnormal condition [23].

Heart rate is very suitable for athletes to know the condition of the heartbeat during exercise whether it is lacking, sufficient, or excessive exercise. Adults who are in good health and are not active or in normal 
resting conditions, the heart rate or pulse is around 60-100 minutes per minute. Heart rate targets are expressed as the range of maximum heart rate percentages that are safe to reach during exercise. The target heart rate of exercise ranges from 50-85 percent of the maximum heart rate, which is usually calculated as the number 220 minus a person's age [24]. Classification of heart rate when working can be seen in table 2 .

Table 2. Classification of working heart rate[25]

\begin{tabular}{|c|c|}
\hline \multicolumn{2}{|c|}{ Heart Rate Work(bpm) } \\
\hline Sitting/Very Light & $60-70$ \\
\hline Light & $70-100$ \\
\hline Moderate & $100-125$ \\
\hline Heavy & $125-150$ \\
\hline Very Heavy & $150-175$ \\
\hline Extremely Heavy & $>175$ \\
\hline
\end{tabular}

Table 2 shows the classification of heart rate values when given a training load. Heart rate in this study was classified into 6 levels, the lowest individual level was $60 \mathrm{bpm}$ and the highest level was more than $175 \mathrm{bpm}$. From the value of the heart rate obtained during exercise, it can be said that the higher the heart rate when given a training load means the organs in the individual work harder.

\subsection{0xygen Saturation}

Oxygen saturation is the presentation of hemoglobin that binds to oxygen in the arteries. Oximeters provide rapid and noninvasive measurement and monitoring techniques to estimate peripheral oxygen saturation (SpO2) hemoglobin, and provide a measure of cardio-respiration function. The use of pulse oximetry also establishes the effectiveness of medical interventions through identified improvements at baseline Sp02 levels and can quickly detect hypoxemia (ie, inadequate amounts of oxygen in the arteries). When healthy people are at rest, the normal condition of SpO2 is above 95\%. However, during high-intensity exercise with maximal oxygen uptake, in certain individuals the value of SpO2 cannot be maintained under normal conditions, dropping below 95\%, which leads to arterial hypoxemia [26]. When oxygen is lacking, the heart rate in athletes may increase to maintain a sufficient supply of oxygen in the body, both at rest and during exercise.

Table 3. Oxygen Saturation Range[26]

\begin{tabular}{|c|c|}
\hline \multicolumn{2}{|c|}{ Sp02 Range(\%) } \\
\hline Low & $70-90$ \\
\hline Medium & $91-94$ \\
\hline High & $95-100$ \\
\hline
\end{tabular}


Table 3 shows the classification of oxygen saturation values in the blood, oxygen saturation conditions are divided into 3 categories low, medium, high. Individuals when exercising oxygen saturation conditions at high levels, it can be said to be in good condition. Mean while, if the oxygen saturation condition is at a low level, then the individual must immediately get medical help.

\subsection{Cardiovascular Load}

Monitoring training loads and match is very important to improve performance and prevent injury. An athlete's performance in responding to training can be estimated from the difference between negative function (fatigue) and positive function (fitness) [7]. Assessment of physical workload can be done based on an increase in work pulse compared to the maximum pulse due to cardiovascular load [9]. Cardio is a term related to the heart and blood vessels which is an important matter for a blood flow in the body. Cardio words itself is short for Cardiovascular Load is an estimation to determine the classification of workload based on the increase in pulse rate of work compared to the pulse rate maximum. In determining the classification of workloads based on the increase in work pulse compared to the maximum pulse rate, cardiovascular load (cardiovascular $=\% \mathrm{CVL}$ ) can be calculated based on the formula below [27]:

$$
\% \mathrm{CVL}=\frac{\text { HRwork-HRrest }}{\text { HRmax }- \text { HRrest }} X 100
$$

\begin{tabular}{|c|c|c|}
\hline Note & $\begin{array}{l}\text { HRwork } \\
\text { HRrest } \\
\text { HRmax }\end{array}$ & $\begin{array}{l}\text { k Heart Rate } \\
\text { ing Heart Rate } \\
\text { mum Heart Rate } \\
\% \text { CVL classification [27] }\end{array}$ \\
\hline & $\%$ CVL & $\begin{array}{c}\text { Classification } \\
\end{array}$ \\
\hline & $<30 \%$ & $\begin{array}{l}\text { No Fatigue } \\
\end{array}$ \\
\hline & $30 \% \mathrm{~s} / \mathrm{d} 60 \%$ & need for improvement (Fatigue Level 1) \\
\hline & $60 \% \mathrm{~s} / \mathrm{d} 80 \%$ & work in no time (Fatigue Level 2) \\
\hline & $80 \% \mathrm{~s} / \mathrm{d} 100 \%$ & urgent action is required (Fatigue Level 3) \\
\hline & $>100 \%$ & no activity allowed \\
\hline
\end{tabular}

Table 4 shows the classification of conditions of cardiovascular workload while exercising. Cardiovascular load conditions are divided into 5 levels, where the higher the percentage of cardiovascular values in an individual when given a training load then the individual is in a state of exhaustion and the exercise program must be stopped to avoid injury.

\subsection{Monitoring System}

Web-based monitoring was chosen in this study, because with a webbased application the application can be seen on a smartphone or on a 
computer. The measurement data on the hardware device will be sent will be uploaded via the wifi network using the ESP8266 wifi module. MQTT broker protocol is used in sending measurement data, because the MQTT broker protocol allows two processes to run together without interdependence, namely the process of monitoring realtime data and the process of storing data on the MongoDB database. The interface of monitoring real-time physiological conditions of athletes can be seen in figure 4 .

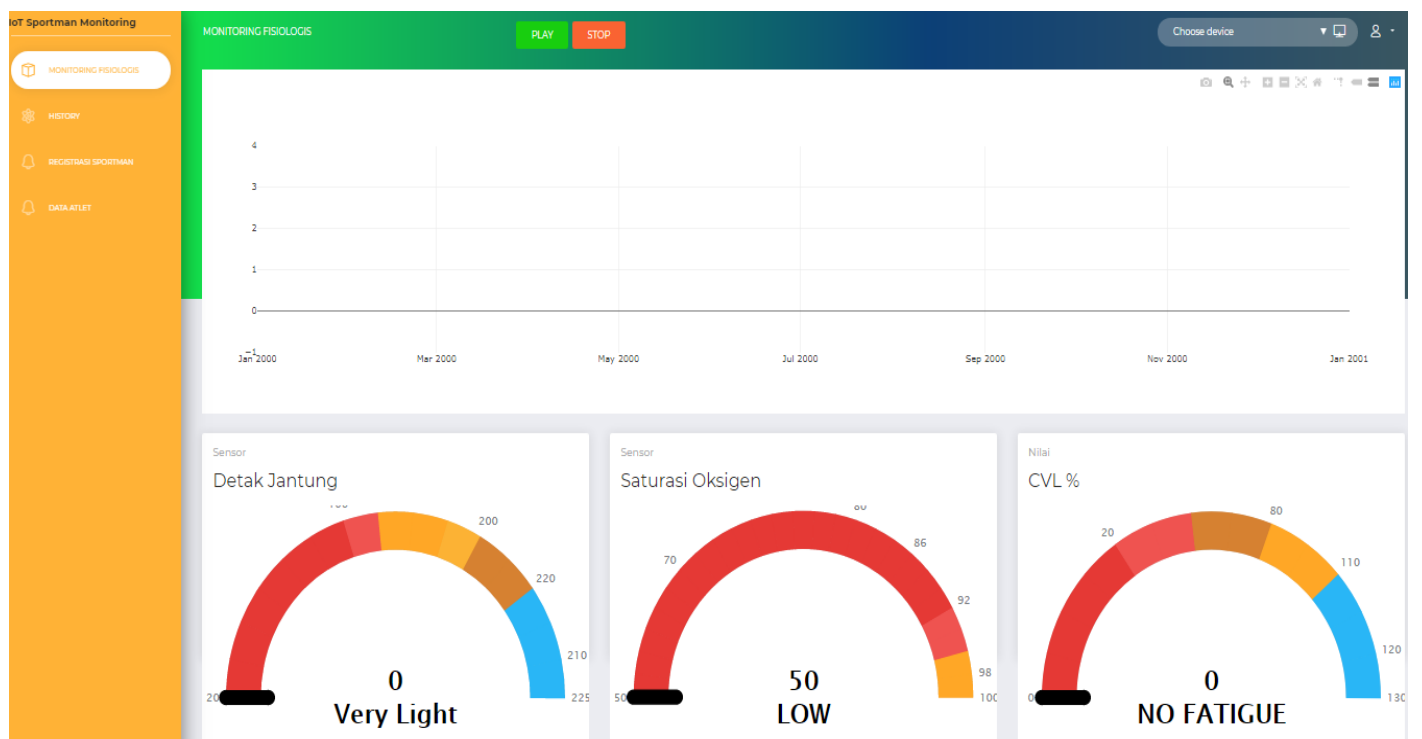

Figure 4. Realtime monitoring interface
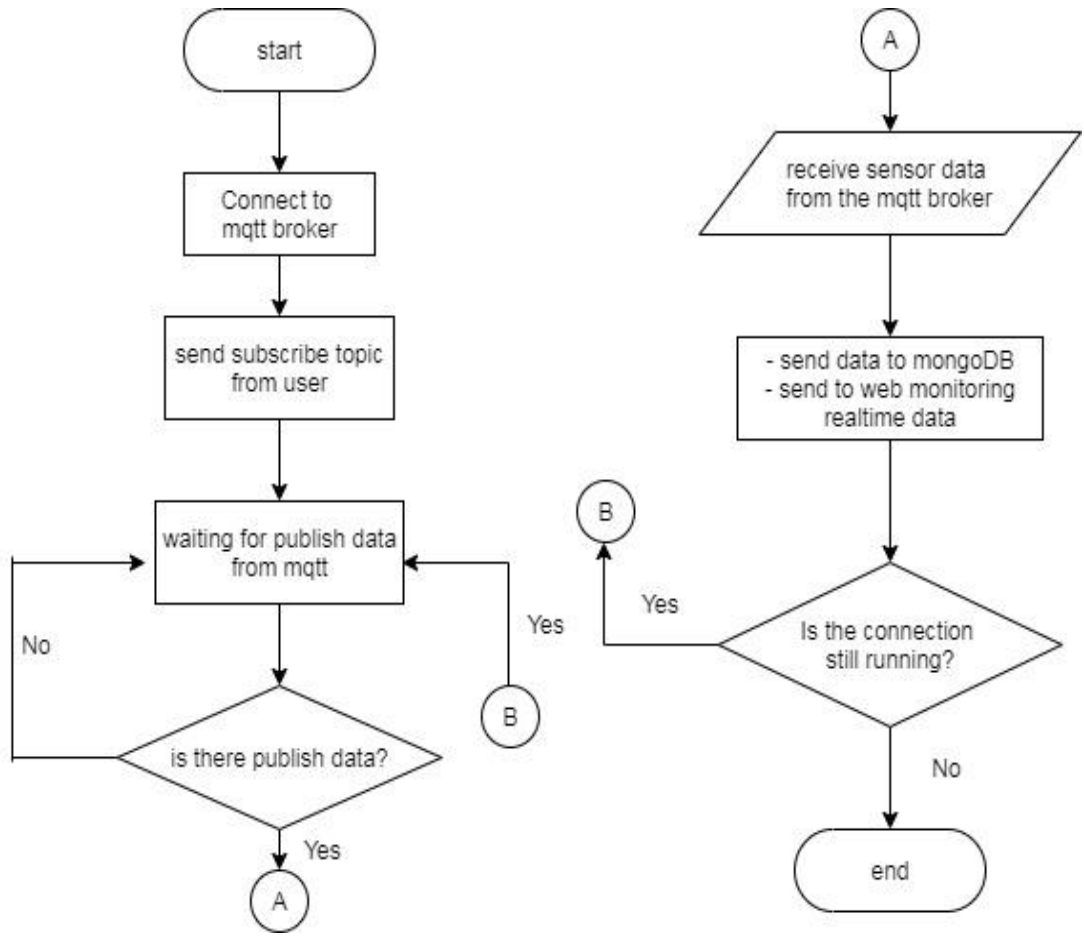

Figure 5. Flowchart process of sending monitoring data 
Figure 5 shows the process of sending data from the sensor to the MQTT broker. The device will send the measurement data when the user requests the data to the MQTT broker by sending a subcribe topic message to MQTT broker. When the data has been successfully sent, then the measurement data will then be displayed on the website in the form of a monitoring data graph, and the data will also be stored in the MongoDB database.

\section{EXPERIMENT AND ANALYSIS}

The main goal of this research is to design a device that can be used automatically and directly monitoring physiologic condition of the user by applying biosensor, and then transmitting the data to the controller and counting the estimation of load cardiovascular level that is experienced. After that, it shows the data result of the measurement in the web application as the information to the user.

To find out how the system developed works, we conducted experiments on a treadmill to monitor runners' physiological conditions. Experiments on the study used 6 volunteers to use hardware on their arms and then run on the treadmill. Hardware installation on the trial participant's arm is shown in Figure 6. The trial participants have different physical conditions, we see information on the physical condition of each participant, namely age, BMI (Body Mass Index), Heart Rate Rest, Heart Rate Max. The physical conditions of each participant in the experiment are shown in table 5 .

Table 5 shows the physical condition of the volunteers who conducted the experiment, the physical conditions that were considered in each individual were age, weight, height, resting heart rate, maximum heart rate, and warming up treatment when before conducting the experiment. The physical condition of the volunteers is very important to know the characteristics of CVL experienced by volunteers in the experiment.

Table 5. Physical Condition of Volunteers

\begin{tabular}{|c|c|c|c|c|c|c|}
\hline User & User 1 & User 2 & User 3 & User 4 & User 5 & User 6 \\
\hline Age(years) & 29 & 19 & 28 & 19 & 32 & 27 \\
\hline $\begin{array}{c}\text { BMI(Height/ } \\
\text { Weight) }\end{array}$ & $175 / 58$ & $173 / 59$ & $174 / 88$ & $170 / 53$ & $160 / 62$ & $160 / 50$ \\
\hline $\begin{array}{c}\text { HR } \\
\text { Rest(bpm) }\end{array}$ & 66 & 96 & 74 & 64 & 81 & 76 \\
\hline $\begin{array}{c}\text { HR } \\
\text { Maks(bpm) }\end{array}$ & 191 & 201 & 192 & 201 & 187 & 193 \\
\hline Note & $\begin{array}{c}\text { Warming } \\
\text { Up }\end{array}$ & $\begin{array}{c}\text { Without } \\
\text { Warming } \\
\text { Up }\end{array}$ & $\begin{array}{c}\text { Without } \\
\text { Warming } \\
\text { Up }\end{array}$ & $\begin{array}{c}\text { Warming } \\
\text { Up }\end{array}$ & $\begin{array}{c}\text { Without } \\
\text { Warming } \\
\text { Up }\end{array}$ & $\begin{array}{c}\text { Without } \\
\text { Warming } \\
\text { Up }\end{array}$ \\
\hline
\end{tabular}




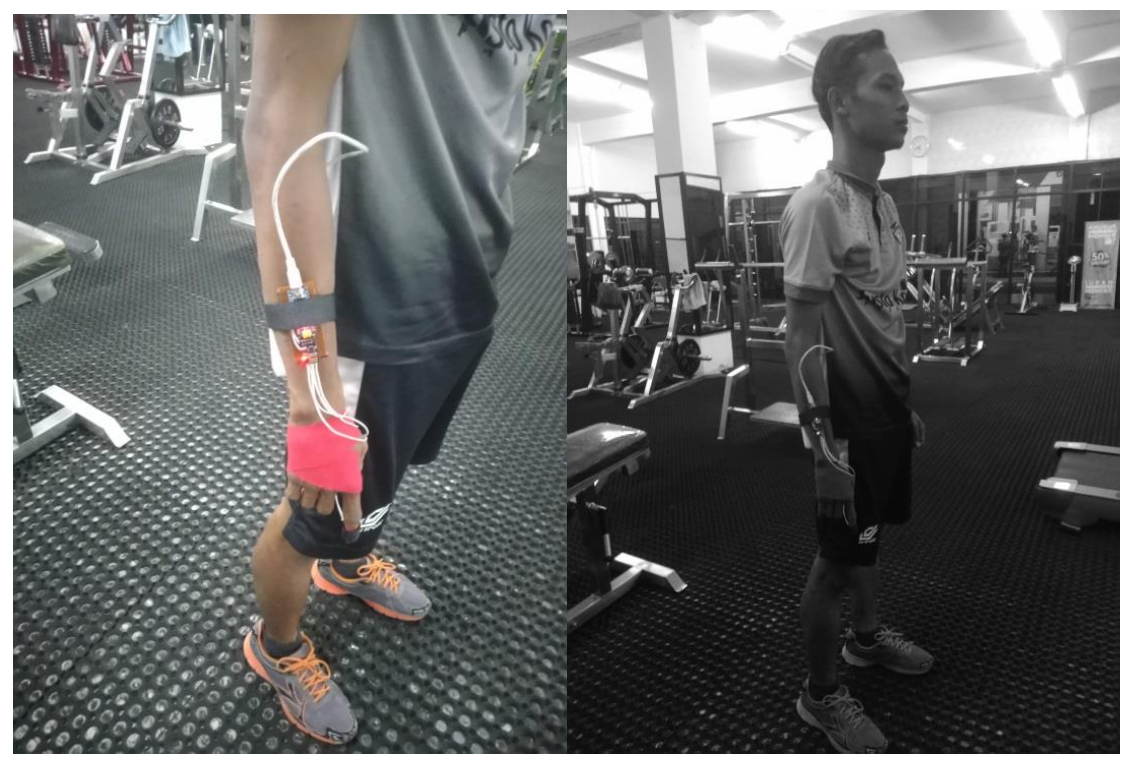

Figure 6. Installation of hardware on the user

Participants do running exercises on the treadmill for 7 minutes divided into 2 weight intensities. The test is divided into two training intensities, namely running at a treadmill speed of $2 \mathrm{~km} / \mathrm{h}$ for 2 minutes and treadmill speed of $8 \mathrm{~km} / \mathrm{h}$ for 5 minutes. In this study changes in speed are used to determine the effect of the intensity of the given training load. With the increase in the intensity of the training load given, there will be inconvenience conditions felt by each individual, but this discomfort will vary in each participant according to their respective fitness conditions. The discomfort felt by the participants was related to the condition of the heartbeat, oxygen saturation and Cardiovascular Load experienced by each participant. The main objective of this study is to monitor the condition of Cardiovascular Load experienced by participants when given a training load. With monitoring of Cardiovascular Load conditions, it is expected to know the level of fatigue and physiological conditions of the participants, so that injury due to exercise overload or overtraining can be avoided. The physiological condition of the participant will be read by the biosensor on the available hardware then the data is transmitted to be displayed on the website to determine the level of physiological conditions of the heartbeat, oxygen saturation and cardiovascular load experienced by the participant. The results of sending sensor reading data on the web application interface in realtime are shown in Figure 7. 


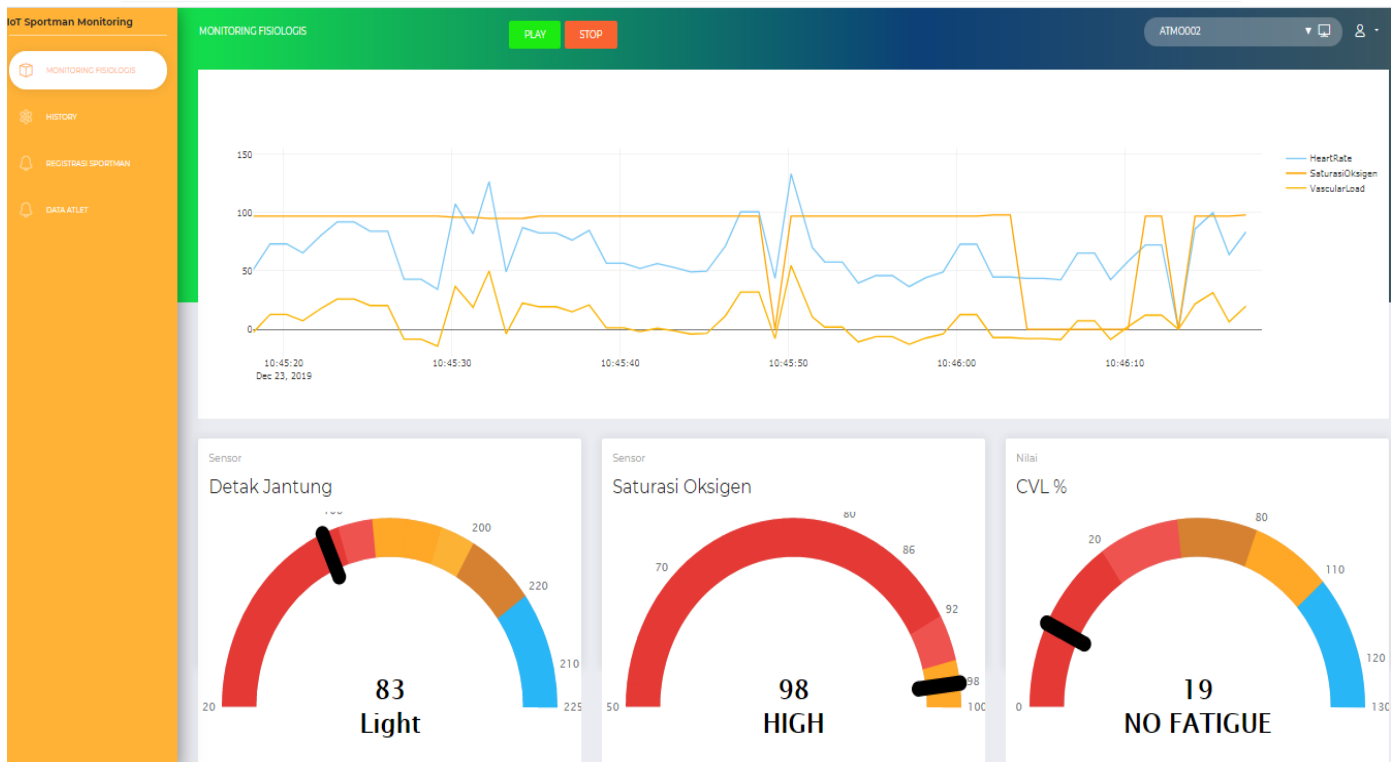

Figure 7. Realtime Monitoring During Exercise

Data on the results of monitoring the condition of the fiological response from 6 participants in an experiment with a treadmill speed of 2 $\mathrm{km} / \mathrm{h}$ are shown in Figure 8,9,10. From the results of the first experiment, Figure 8 shows a maximum HRwork of user- 1 at 84 bpm, user- 2 at $125 \mathrm{bpm}$, user- 3 at 99 bpm, user- 4 at 80 bpm, user- 5 at 113 bpm and for user-6 at 103 $\mathrm{bpm}$. If seen from the limits of the values in table 2 , it can be seen that when given treatment on a treadmill with a speed of $2 \mathrm{~km} / \mathrm{h}$, for user- 1 , user- 3 and user- 4 belong to the Light category while for user- 2 , user -5 and user -6 belong to the Moderate category.

Figure 9 shows the oxygen saturation conditions in each user when treated in the first experiment. Figure 9 shows the oxygen saturation conditions for user-1 at 96 (\%), user-2 at 97 (\%), user-3 at 96-97 (\%), user-4 at $97(\%)$, user-5 at $97(\%)$, while user-6 is $96(\%)$. Then the oxygen saturation value in four users is in the range of values of 95-100 (\%) or can be said to be in a High (Good) condition.

Figure 10 shows the user's CVL condition when given the first try. Figure 10 shows that the highest CVL value for user- 1 is $14 \%$, for user- $226.92 \%$, for user-3 29.50\%, for user $411.67 \%$, for user-5 30,84\% and user- 6 at the value of $23.07 \%$. Based on table 4, the CVL values of users 1,2,3,4 and 6 are in the range of less than $30 \%$ so they can be categorized at the No Fatigue level. Then for CVL condition on user 5 is at Fatigue Level 1. 


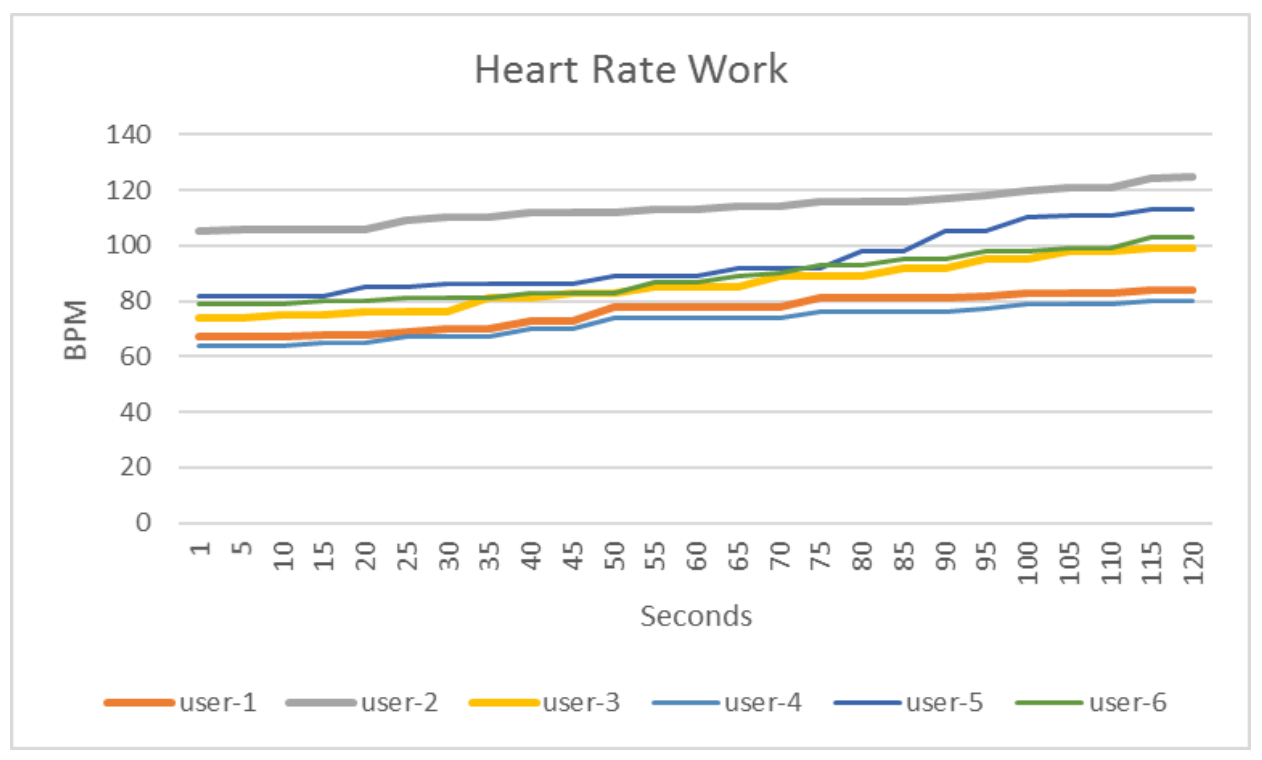

Figure 8. Heart Rate Work ( Treadmill $2 \mathrm{~km} / \mathrm{h}$ )

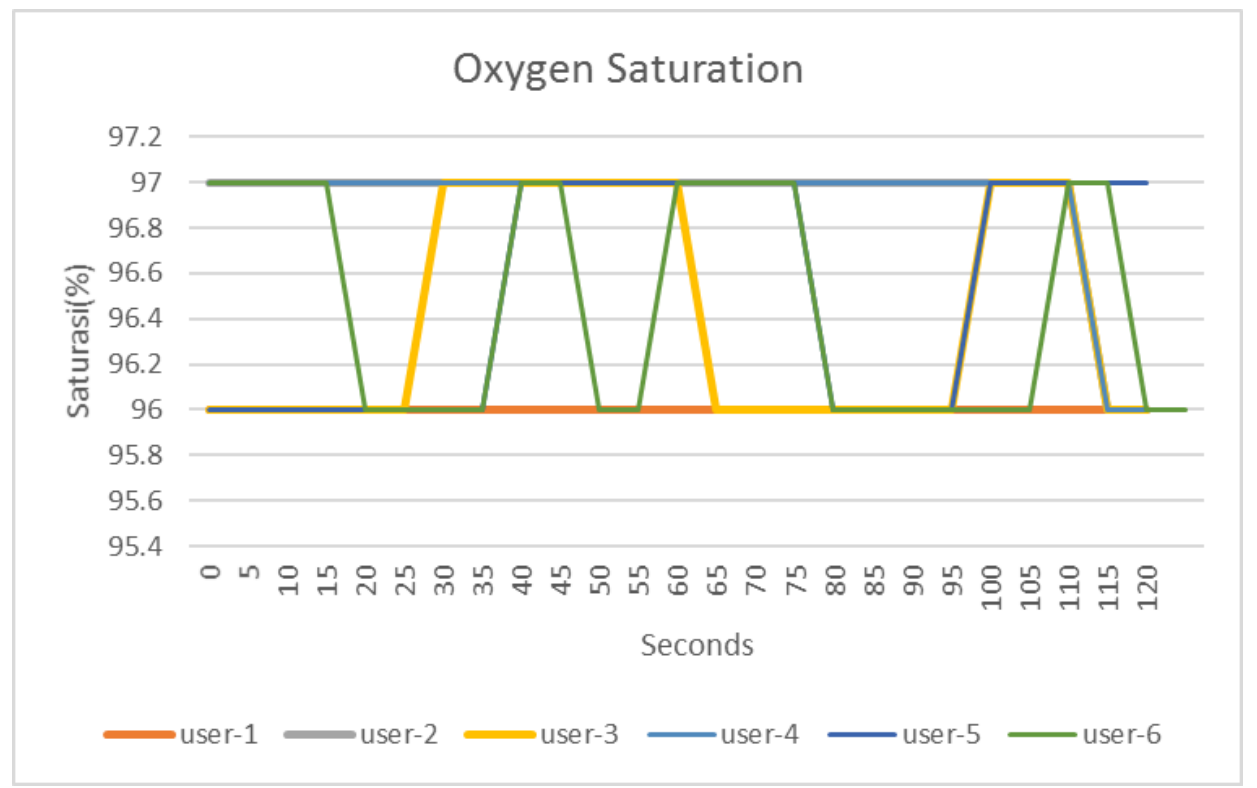

Figure 9. Oxygen Saturation ( Treadmill $2 \mathrm{~km} / \mathrm{h}$ ) 


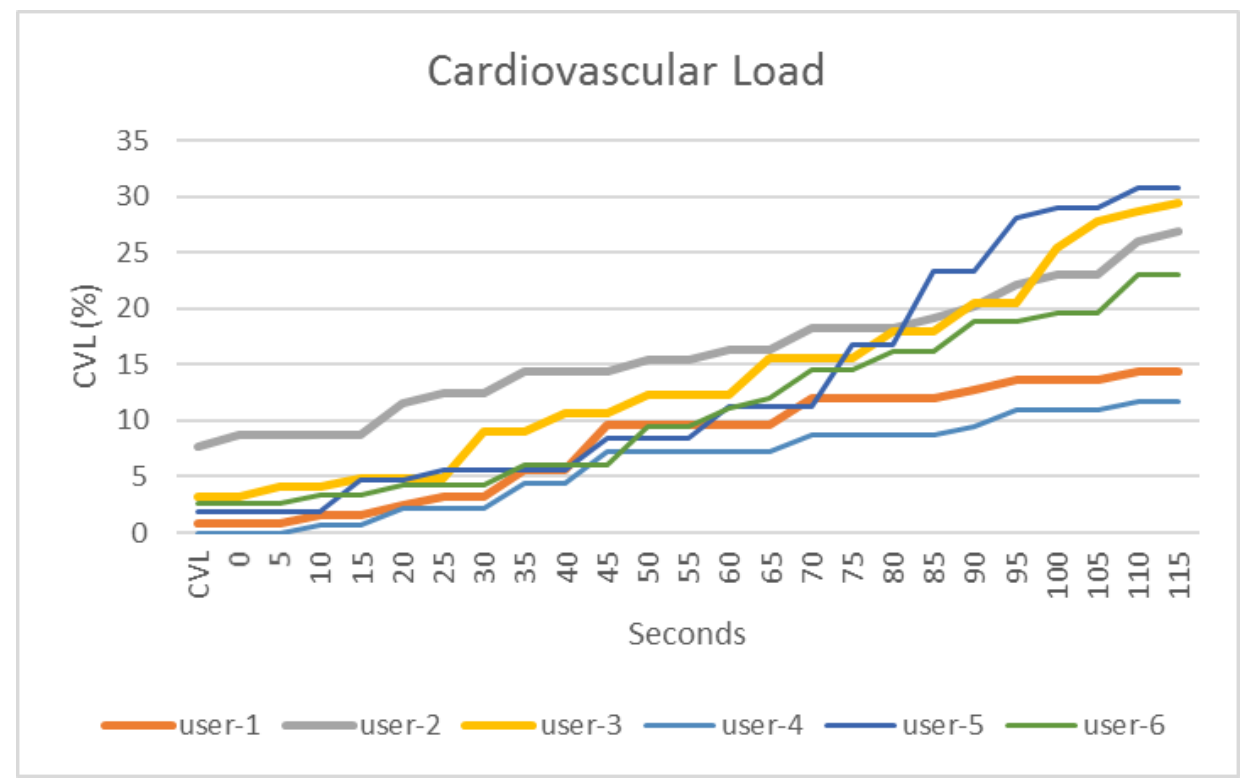

Figure 10. CVL ( Treadmill $2 \mathrm{~km} / \mathrm{h}$ )

Figures 11, 12, 13 are user results when given a second load treatment with a treadmill speed of $8 \mathrm{~km} / \mathrm{h}$. Figure 11 shows the HRwork condition of all users when given a training load. Figure 11 shows the maximum HRwork experienced by user- 1 is 132 bpm, so user- 1 is included in the Heavy category. User-2 HRwork is $170 \mathrm{bpm}$ and User-3 HRwork is 158, so user-2 and user-3 heart rates are included in the Very Heavy category. Then for user- 4 at a value of $130 \mathrm{bpm}$, user-4 is in the Heavy category. While HRwork for user-5 is $174 \mathrm{bpm}$ and user- 6 is $156 \mathrm{bpm}$, so user- 5 and user- 6 are in the Very Heavy category.

Figure 12 shows the user's oxygen saturation condition when given a training load with a treadmill speed of $8 \mathrm{~km} /$ hour. Figure 12 shows the oxygen saturation value in 6 users is at a fluctuating value with the lowest value from 95 to the highest 98 for all three users. But even though the oxygen saturation value of four users fluctuates, the oxygen saturation value of four users is included in the High category or can be said to be in good condition. This fluctuating condition can be caused by the respiratory conditions of volunteers who have been breathing a lot so that oxygen uptake is less than optimal and irregular.

Figure 13 shows the Cardiovascular Load value experienced by users with a treadmill speed of $8 \mathrm{~km} / \mathrm{h}$. Figure 13 shows the value of cardiovascular load experienced by user- 1 is at a value of $52 \%$, in other words the value of cardiovascular load for user-1 is included in the category of Fatigue Level 1. Then for user- 2 the cardiovascular load experienced is at $68 \%$, while for user3 cardiovascular load is $72 \%$. Then the cardiovascular values of user- 2 and user-3 fall into the Fatigue Level 2 category. While the cardiovascular burden experienced by user- 4 is at a value of $49 \%$, in other words the cardiovascular burden value for user-4 falls into the Fatigue Level Category 1. Furthermore 
for user-5 the CVL condition is at $93.04 \%$ and user- 6 is at a value of $80.82 \%$, then user-5 and user 6 fall into the Fatigue Level 3 category.

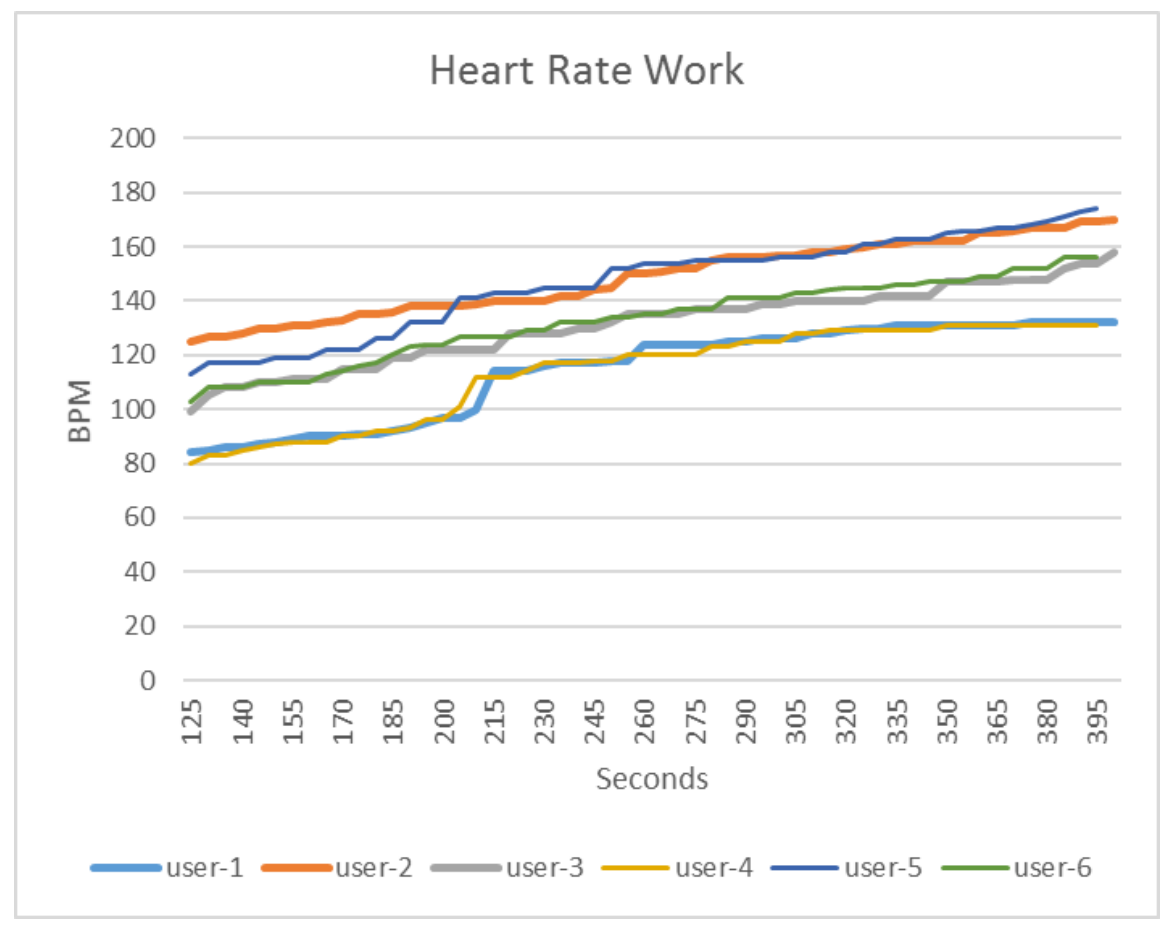

Figure 11. Heart Rate Work ( Treadmill $8 \mathrm{~km} / \mathrm{h}$ )

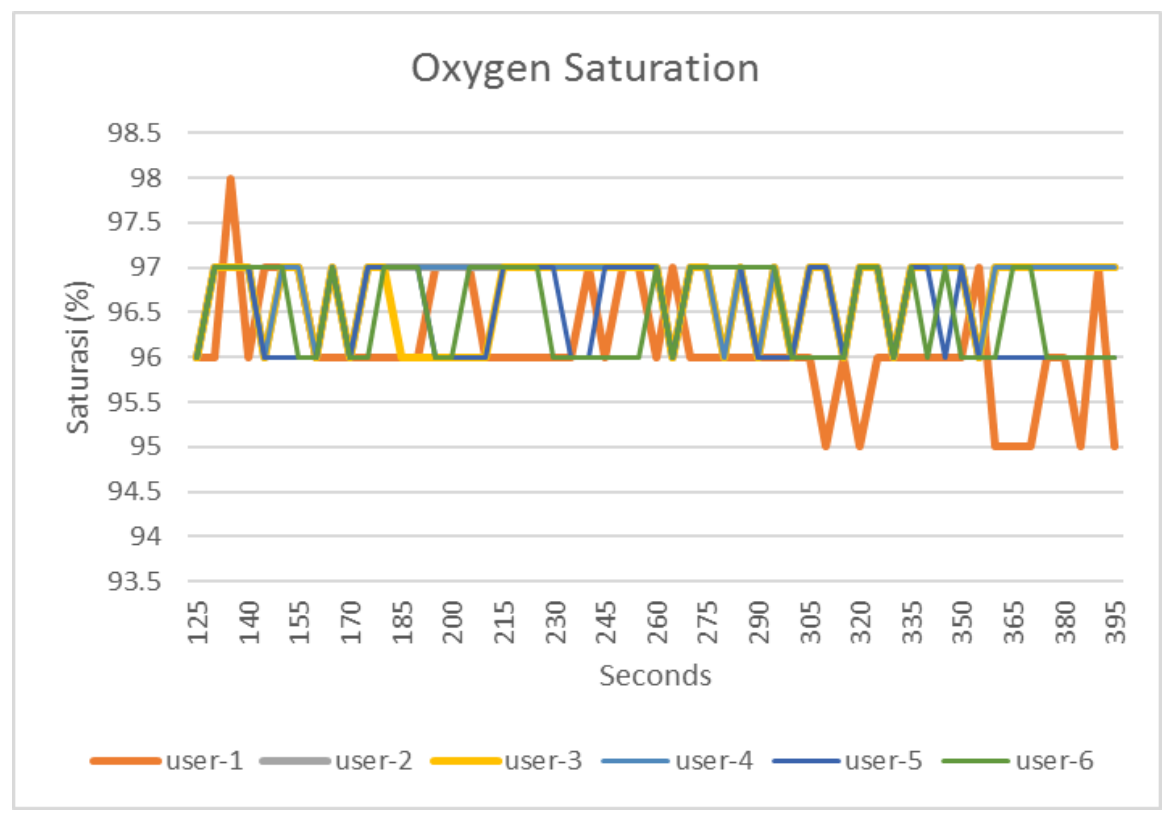

Figure 12. Oxygen Saturation ( Treadmill $8 \mathrm{~km} / \mathrm{h}$ ) 


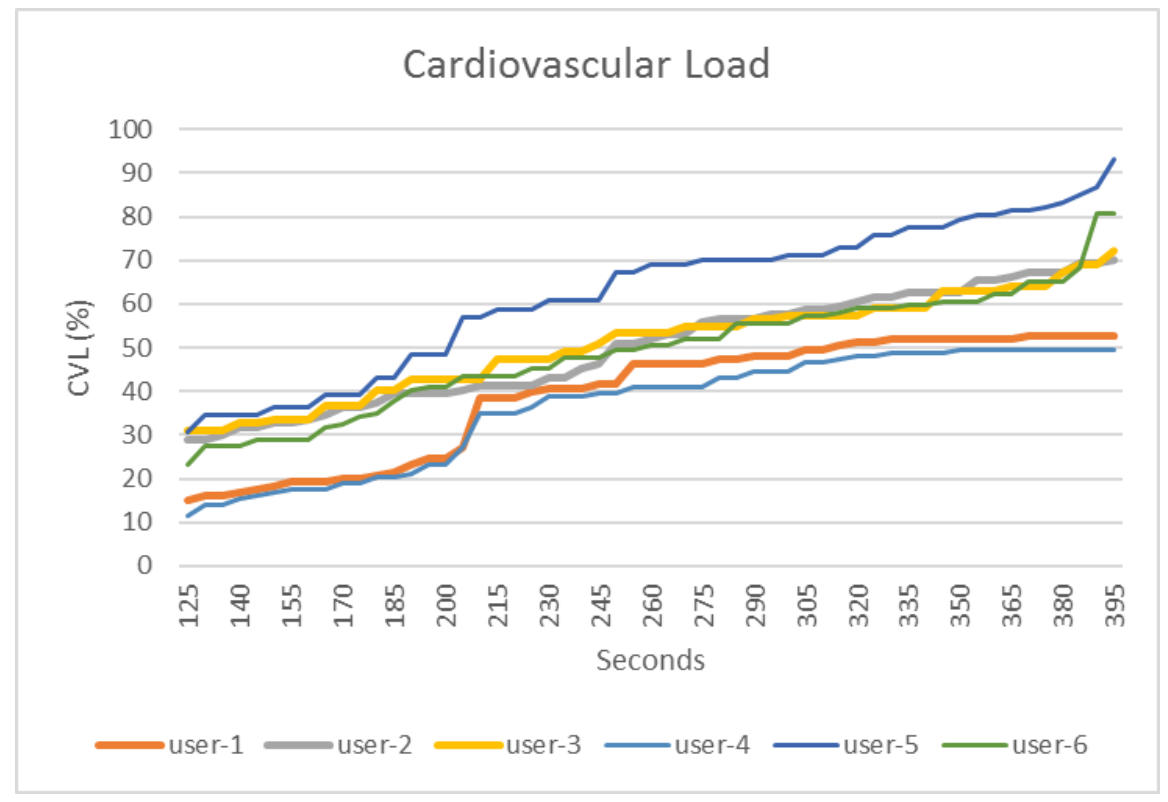

Figure 13. CVL ( Treadmill $8 \mathrm{~km} / \mathrm{h}$ )

\subsection{Heart Rate dan Saturasi Oksigen}

To know the effectiveness of the sensors we used in this research with Pulse Oximeter (Figure 14) healthiness product that available on the commercial to measure the condition of heart beat and oxygen saturation. The results of comparison for heart rate show that the error obtained is less than $1 \mathrm{bpm}$, so it can be confirmed that the sensor we use can be used to measure heart rate. The measurement of heart beat on the sensors that we used is compared with Pulse Oximetry shows in table 6.

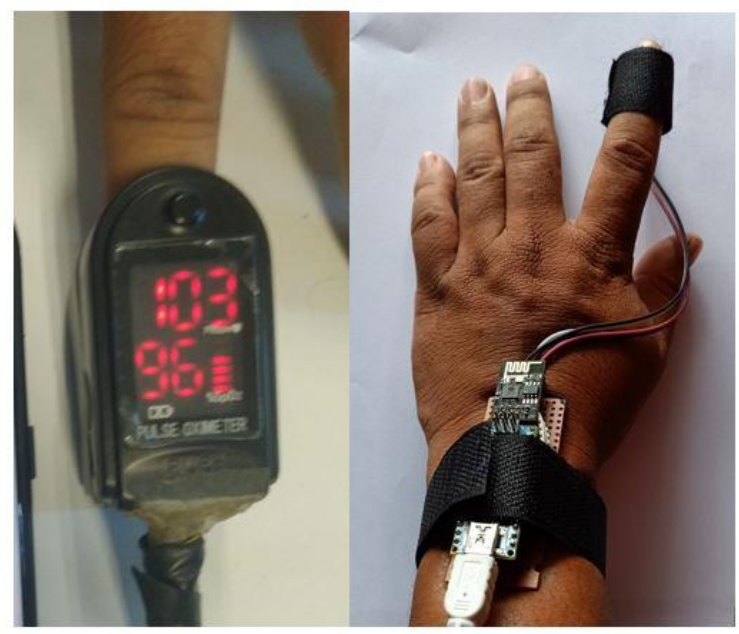

Figure 14. Comparison with Pulse Oximetry 
Table 6. Comparison between heart rates measured with max30100 and Pulse Oximetry

\begin{tabular}{|c|c|c|c|}
\hline Test number & Sensor max 30100 & Pulse oximetry & Error \\
\hline 1 & 101 & 101 & 0 \\
\hline 2 & 94 & 93 & 1 \\
\hline 3 & 103 & 102 & 1 \\
\hline 4 & 95 & 95 & 0 \\
\hline 5 & 96 & 95 & 1 \\
\hline 6 & 98 & 98 & 0 \\
\hline 7 & 91 & 91 & 0 \\
\hline Average & & & 0,42 \\
\hline
\end{tabular}

Then the oxygen saturation in the blood, the comparison of the sensors we use with Pulse Oximetry shows below an average error of 0.14 . Thus the device we use can be used to measure oxygen saturation, the comparison error table is shown in table 7.

Table 7. Comparison between oxygen saturation measured with max30100 and Pulse Oximetry

\begin{tabular}{|c|c|c|c|}
\hline Test number & Sensor max 30100 & Pulse oximetry & Error \\
\hline 1 & 96 & 96 & 0 \\
\hline 2 & 97 & 97 & 0 \\
\hline 3 & 97 & 96 & 1 \\
\hline 4 & 96 & 96 & 0 \\
\hline 5 & 96 & 96 & 0 \\
\hline 6 & 98 & 98 & 0 \\
\hline 7 & 97 & 97 & 0 \\
\hline Average & & & 0.14 \\
\hline
\end{tabular}

\section{CONCLUSION}

For an athlete fitness is an important thing that must be maintained at all times, so that they are always ready to face a race or match. Good and proper exercise will maintain the fitness condition of an athlete, but excessive exercise will cause athletes in a condition of overtraining or excessive fatigue or even injury. In this study proposes an application for monitoring the condition of the training load experienced by the athlete's body which is manifested in the value of the Cardiovascular Load. We use an oximetry sensor to monitor physiological information from athletes, through a microcontroller data collected from heart rate monitoring and oxygen saturation. Data is sent to a web-based application to be displayed in realtime and calculated the level of physiological conditions experienced by athletes when training. With this application allows exercises given in accordance with the conditions of each athlete so that fitness can be maintained. From the experimental results, the developed application can monitor the physiological condition trends of runners when given the intensity of the 
exercise, and the applications developed can provide information on the physiological level experienced by runners.

For example, what happens to users 2 and 4, they have almost the same physical condition characteristics. But when they are given the same training load, the body's response and the level of training load felt by user- 1 and user- 2 are different. So when given a training load, user-1 is in the Fatigue Level 1 category and user- 2 is in the Fatigue Level 2 category.

From this study, it can be seen that the sensors we use can measure heart rate and oxygen saturation with errors for each of them at the values of 0.42 and 0.14 . For further work, the addition of sensors to obtain other physiological information can be done to get more maximum results, for example the addition of sweat sensors to determine the level of dehydration might be able to add information on runner conditions. In this work only monitoring to determine the level of physiological conditions experienced by runners. Addition of exercise recommendations that must be done to maintain fitness may be added to further work.

\section{Acknowledgment}

This research was supported in part by Ministry of Research, Technology and Higher Education of Indonesia, under scheme 'Penelitian Unggulan Perguruan Tinggi (PTUPT)', Grant No. B/87/E3/RA.00/2020.

\section{REFERENCES}

[1] S. Kodama et al., Cardiorespiratory fitness as a quantitative predictor of all-cause mortality and cardiovascular events in healthy men and women: A meta-analysis, JAMA - J. Am. Med. Assoc., vol. 301, no. 19, pp. 2024-2035, 2009.

[2] T. W. Calvert, E. W. Banister, M. V. Savage, and T. Bach, A Systems Model of the Effects of Training On Physical Performance, Aust J Sport. Med, vol. SMC-6, no. 2, pp. 94-102, 1976.

[3] R. Pind and J. Mäestu, Monitoring training load: necessity, methods and applications, Acta Kinesiol. Univ. Tartu., vol. 23, p. 7, 2018.

[4] B. T. Hulin, T. J. Gabbett, D. W. Lawson, P. Caputi, and J. A. Sampson, The acute: Chronic workload ratio predicts injury: High chronic workload may decrease injury risk in elite rugby league players, Br. J. Sports Med., vol. 50, no. 4, pp. 231-236, 2016.

[5] R. H. Morton, Modelling training and overtraining, J. Sports Sci., vol. 15, no. 3, pp. 335-340, 1997.

[6] C. R. Bellenger, R. L. Thomson, P. R. C. Howe, L. Karavirta, and J. D. Buckley, Monitoring athletic training status using the maximal rate of heart rate increase, J. Sci. Med. Sport, vol. 19, no. 7, pp. 590-595, 2016.

[7] J. G. Dong, The role of heart rate variability in sports physiology (Review), Exp. Ther. Med., vol. 11, no. 5, pp. 1531-1536, 2016. 
[8] J. E. Wingo, M. S. Ganio, and K. J. Cureton, Cardiovascular drift during heat stress: Implications for exercise prescription, Exerc. Sport Sci. Rev., vol. 40, no. 2, pp. 88-94, 2012.

[9] M. Gleeson, Temperature regulation during exercise: Directions1983, Int. J. Sports Med., vol. 19, no. 2, pp. S96-S99, 1998.

[10] N. S. A. Zulkifli, F. K. Che Harun, and N. S. Azahar, XBee wireless sensor networks for Heart Rate Monitoring in sport training, 2012 Int. Conf. Biomed. Eng. ICoBE 2012, no. in C, pp. 441-444, 2012.

[11] K. Malhi and S. C. Mukhopadhyay, A Zigbee-Based Wearable Physiological Parameters Monitoring SystemA Zigbee-Based Wearable Physiological Parameters Monitoring System, IEEE Sens. J., vol. 45, no. 8, pp. 799-804, 2012.

[12] J. Segura-Garcia, M. Garcia-Pineda, M. Tamarit-Tronch, R. M. Cibrian, and R. Salvador-Palmer, Cost-effective eHealth system based on a multi-sensor system-on-chip platform and data fusion in cloud for sport activity monitoring, Electron., vol. 7, no. 9, pp. 1-13, 2018.

[13] Z. Ovadia-blechman, O. Gino, L. Dandeker, and N. Sheffer, The Feasibility of Flat, Portable and Wireless Device for Non-Invasive Peripheral Oxygenation Measurement over the Entire Body, J. Biomed. Sci. Eng., no. March, pp. 147-159, 2016.

[14] A. Ahmed, A. A. Lukman, A. James, O. O. Mikail, B. U. Umar, and E. Samuel, Human Vital Physiological Parameters Monitoring: A Wireless Body Area Technology Based Internet of Things, J. Teknol. dan Sist. Komput., vol. 6, no. 3, p. 115, 2018.

[15] S. T. Chen, S. S. Lin, C. W. Lan, and H. Y. Hsu, Design and development of awearable device for heat stroke detection, Sensors (Switzerland), vol. 18, no. 1, 2018.

[16] L. Djaoui, M. Haddad, K. Chamari, and A. Dellal, Monitoring training load and fatigue in soccer players with physiological markers, Physiol. Behav., vol. 181, no. September, pp. 86-94, 2017.

[17] S. L. Halson, Monitoring Training Load to Understand Fatigue in Athletes, vol. 44, 2014.

[18] Subono, M. U. H. Al Rasyid, and I. G. P. Astawa, Implementation of Energy Efficiency Based on Time Scheduling to Improve Network Lifetime in Wireless Body Area Network (WBAN), Emit. Int. J. Eng. Technol., vol. 3, no. 2, pp. 28-42, 2016.

[19] R. T. Li, S. R. Kling, M. J. Salata, S. A. Cupp, J. Sheehan, and J. E. Voos, Wearable Performance Devices in Sports Medicine, Sports Health, vol. 8, no. 1, pp. 74-78, 2016.

[20] Y. Khan, A. E. Ostfeld, C. M. Lochner, A. Pierre, and A. C. Arias, Monitoring of Vital Signs with Flexible and Wearable Medical Devices, Adv. Mater., vol. 28, no. 22, pp. 4373-4395, 2016.

[21] D. Dias and J. P. S. Cunha, Wearable health devices-vital sign monitoring, systems and technologies, Sensors (Switzerland), vol. 18, no. 8, 2018. 
[22] D. Chong and N. Zhu, Human heat acclimatization in extremely hot environments: A review, Procedia Eng., vol. 205, pp. 248-253, 2017.

[23] J. Y. Khan and M. R. Yuce, Wireless Body Area Network(WBAN) for Medical Applications, J. Med. Syst., vol. 36, no. 3, pp. 1441-1457, 2012.

[24] L. E. Armstrong, D. J. Casa, M. Millard-Stafford, D. S. Moran, S. W. Pyne, and W. O. Roberts, Exertional heat illness during training and competition, Med. Sci. Sports Exerc., vol. 39, no. 3, pp. 556-572, 2007.

[25] D. Dubeau, A. Kolus, D. Imbeau, and P. Dub, Classifying work rate from heart rate measurements using an adaptive neuro-fuzzy inference system, vol. 54, pp. 158-168, 2016.

[26] R. P. Garrido-Chamorro, M. González-Lorenzo, J. Sirvent-Belando, C. Blasco-Lafarga, and E. Roche, Desaturation patterns detected by oximetry in a large population of athletes, Res. Q. Exerc. Sport, vol. 80, no. 2, pp. 241-248, 2009.

[27] D. P. Restuputri, A. K. Pangesti, and A. K. Garside, The measurement of Physical Workload and Mental Workload Level of Medical Personnel, J. Tek. Ind., vol. 20, no. 1, p. 34, 2019. 